\title{
Rain Streaks Detection and Removal in Image based on Entropy Maximization and Background Estimation
}

\author{
Ruchi katre \\ Surabhi Group of Institution \\ Bhopal, India
}

\author{
Nitesh Dodkey \\ Surabhi Group of Institution \\ Bhopal, India
}

\begin{abstract}
The rain removal from an image in the rainy season is also a required task to identify the object in it. It is a challenging problem and has been recently investigate extensively. In this paper the entropy maximization and background estimation based method is used for the rain removal. This method is based on single-image rain removal framework. The raindrops are greatly differing from the background, as the intensity of rain drops is higher the background. The entropy maximization is very much suitable for the rain removal. Experimental results express the efficacy of the rain removal by proposed algorithm is better than the method based on saturation and visibility features.
\end{abstract}

\section{Keywords}

Dictionary learning, image decomposition, morphological component analysis (MCA), rain removal, sparse representation

\section{INTRODUCTION}

Removal of rain streaks has recently received much useful for object identification in rainy images. There are the lots of topics that the researcher focuses that cover the field of image and signal processing. The field extends from the basic level first the basic images are improved and then the images in the bad weather as the rain, snow or fog (or haze) etc. The removal of rain streaks has recently received much attention [1-6] in the research work in the field of image processing. The rain removal is just like the image enhancement and may come in the category of image noise removal or image restoration. Garg et al [2] in 2004 worked on dynamics of correlation based rain droplets detection and removal in videos. In the next subsequent year 2005 author shown in [3] that by altering some camera parameters as exposure time and depth of field the appearance can be enhanced and mitigate the effects of rain without the scene appearance alteration. Furthermore, Barnum et al. [1] presented model of single image rain or snow streak detection. Bossu et al. [7], presented a selection rules based on photometry. The photometry and size are used in selection rules to select the latent rain streaks in a video, in which the rain streaks orientations histogram is estimated with computed geometric moments. Meanwhile some researchers [8-10] focused on raindrop recognition in images or videos that is different from rain streaks detection.

The rain and non-rain parts in a single image are very closely mixed up and the identification of rain streaks is not an easy task. In this paper, we compare a single-image rain streak removal based on morphological component analysis (MCA) by decomposition of rain streaks [11]-[12]. The signal and image processing for the filtering and region specification are discussed in the previous works [13-22]. In this method, a bilateral filter is applied for an image to decompose it into the low-frequency (LF) and high-frequency (HF) parts. The HF part is then decomposed into rain component and non-rain component by performing sparse coding and dictionary learning on MCA. While the saturation and visibility feature (SVF) based rain removal uses high pass filter and the orientation filter [23].

The rest of this paper is organized as follows. In Section II, explain saturation and visibility feature (SVF) based image de-raining. Section III presents the image decomposition, sparse coding, and dictionary learning techniques for singleimage-based rain streak removal framework. In Section IV, experimental results are demonstrated. Finally, Section V concludes this paper.

\section{METHOD BY SOO-CHANG PEI}

Raindrops and snowflakes are very bright dots and streaks spreading in the photograph [17]. To remove the rain and snow, we need to enhance the signal of raindrops and snowflakes for the purpose of raindrops and snowflakes detection. To enhance the signal contained in the color image, we multiply the visibility layer and the inverse of the saturation layer pixel by pixel to obtain the initial potential candidate visibility pixel of the snow or rain.

$$
R_{i n i}=(1-S) * V
$$

where $S$ and $V$ represent the saturation and visibility in $H S V$ color model [8], respectively. As from the study the power spectral distribution of rain and snow "streaks" in the 2D frequency space is elliptical and orientated with certain direction, but rain drop is random and not orientated to any directions. Hence we need different model to process. In this method the basic concept is object detection and removal. For the purpose of object detection, we need to enhance the signal of the object in the image to improve the correctness of detecting object. The flow chart of the proposed rain removal method is showed in Fig.1. Using these fundamental concepts, the proposed method will be introduced in the following subsections.

\subsection{Rain Heightening and Detection}

The properties of rain and snow have high visibility and low saturation. Thus, we exploit these unique properties of rain and snow to detect the candidates of raindrop and snow pixels.

1. Merge Saturation and Visibility

2. High Pass Filter

3. Orientation Filter: According that the raindrops drop from the top to the bottom, the horizontal streaks will not be the raindrops no matter how strong the wind is. Use a high pass filter in the horizontal direction to filter out the streaks which are not raindrops. By this step, we can get a gray image which has enhanced the raindrops. 
4. Threshold: By extensive experiments, we can get the more accurate images of rain or snow by selecting the intensity of top 10 to 15 percent. Hence we select the average value, top 13 percent.

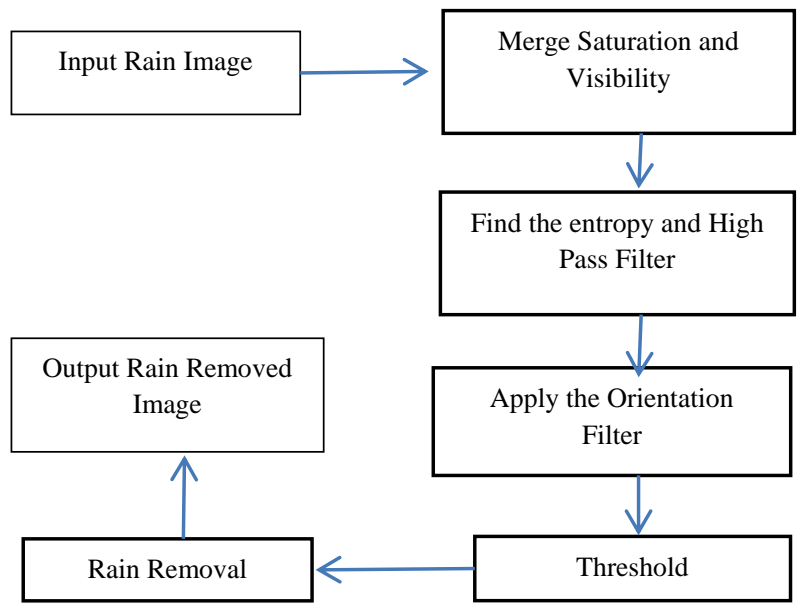

Fig. 1: flow chart of the saturation feature based rain removal method

\subsection{Rain Removal}

After select the raindrop pixels in the photograph, we need to remove these raindrops by in painting. Fig 3 is an example of the in painting process. The high intensity of image and the red region is actually the rain pixel. We assume the rain streak region is marked as "1" and we use the background region (yellow region) to fill the rain region (red region) with average surrounding background value. The simulation of recovered clear image with rain removal as shown in Fig 2(b) this process do in both saturation and visibility layers.

\section{DECOMPOSITION FRAMEWORK}

Based on the two dictionaries $D_{H F-R}$ and $D_{H F-G}$, we perform sparse coding by applying the OMP algorithm [13] [22] for each patch $b_{H F}^{K}$ extracted from $I_{H F}$ via minimization of (5) to find its sparse coefficients $\theta_{H F}^{K}$. Different from traditional MCA algorithms, where sparse coding and dictionary learning should be iteratively performed, we perform sparse coding only once for each patch $b_{H F}^{K}$ with respect to $D_{H F}=$ $\left[D_{H F-R} D_{H F-G}\right]$. Then, each reconstructed patch $b_{H F}^{K}$ can be used to recover either geometric component $I_{H F}^{G}$ or rain component $I_{H F}^{R}$ of $I_{H F}$ based on the sparse coefficients $\theta_{H F}^{\sim K}$ as follows. We set the coefficients corresponding to in to zeros to obtain, whereas the coefficients corresponding $D_{H F-G}$ to $\theta_{H F}^{\sim K}$ in to zeros to obtain. Therefore, each patch $b_{H F}^{K}$ can be re-expressed as either $b_{H F-G}^{K}=D_{H F-G} \times \tilde{\theta}_{H F-G}^{K}$ or $\tilde{b}_{H F-R}^{K}$ $=D_{H F-R} \times \tilde{\theta}_{H F-R}^{K}$, which can be used to recover $I_{H F}^{R}$ or $I_{H F}^{G}$, respectively, by averaging the pixel values in overlapping regions. Finally, the rain-removed version of the image I can be obtained via $I^{\text {Non-Rain }}=I_{L F}+I_{H F}^{G}$, as illustrated in Fig. 4(e).

\section{PROPOSED METHOD}

The proposed method find the rain drops from the orientation filter and after the orientation filtering the entropy maximization based remaining rain drops are detected and the background is estimated for the rain removal. The step by step procedure is explained in the figure 2 .

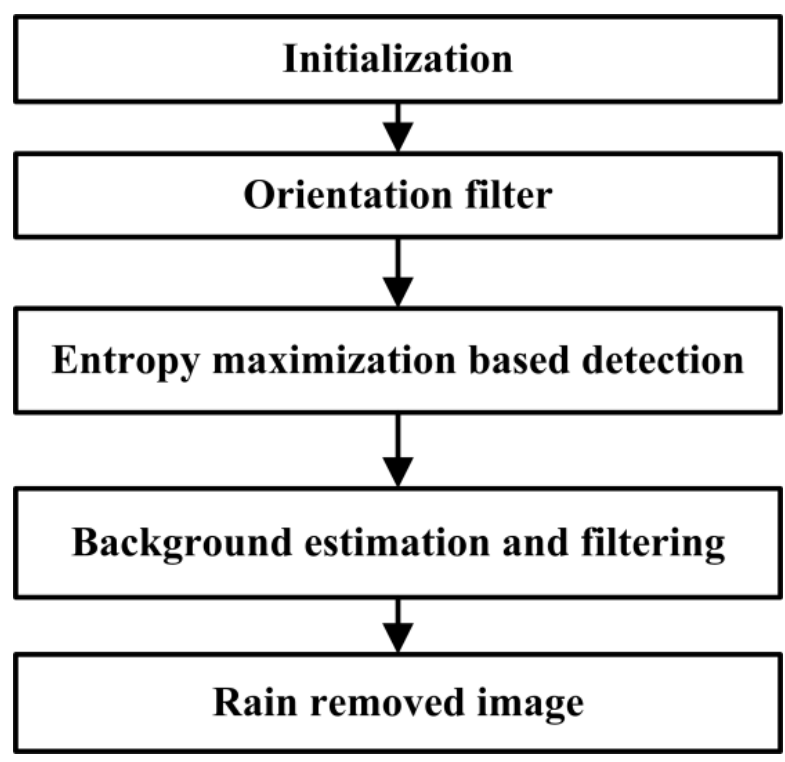

Fig. 2. The flowchart of the proposed method

The decomposition based rain removal is based on the orientation of the rain drops. The vertically oriented [13, 22] rain drops are separated by the decomposition base method $[23,24]$.

The Entropy Maximization Algorithm [25-27] based residual raindrop detection is performed as: Consider the system of equations $A x=b$, where $A$ is a $n \times m$ nonnegative matrix, and b $b \in R^{n}$ is the noisy nonnegative data vector. If the modeling and the data were exact, the system would have a nonnegative solution. In light of the noisy data and the modeling, the sense in which the system $A x=b$ is to be solved is that of minimum negative cross log entropy (also known as negative cross Burg entropy, or Itakura-Saito distance), i.e., by solving

$$
\begin{array}{r}
\min \operatorname{imizeL}(x)=\sum_{i=0}^{\text {def }} \sum^{n}-\log \frac{b_{i}}{[A x]_{i}}+\frac{b_{i}}{[A x]_{i}} \\
\text { subject to } x \geq 0
\end{array}
$$

The first thing to note is that if $x^{*}$ satisfies $A x^{*}=b$, and then $x=x *$ solves the problem. The second thing to note is that $L(x)$ is not a convex functional of $x$, since the logarithmic term is concave. We look at two related algorithms, as follows.

LogEnt I:

$$
x_{j}^{k=1}=x_{j}^{k} \sqrt{\frac{\left[A^{T} p^{k}\right]_{j}}{\left[A^{T} q^{k}\right]_{j}}}, \quad j=1,2, \ldots . m
$$

And LogEnt II:

$$
x_{j}^{k=1}=x_{j}^{k} \cdot \frac{\left[A^{T} p^{k}\right]_{j}}{\left[A^{T} q^{k}\right]_{j}}, \quad j=1,2, \ldots . m
$$


Where

$$
\begin{gathered}
p_{i}^{k}=\frac{b_{i}}{\left(\left[A x^{k}\right]_{i}\right)^{2}}, \\
q_{i}^{k}=\frac{1}{\left[A x^{k}\right]_{i}}, \quad i=1,2, \ldots n .
\end{gathered}
$$

If $x^{1}$ has positive components, then all the future $x^{k}$ have all positive components as well. So expressions like $x_{j}^{k+1} / x_{j}^{k}$ are always meaningful. We denote the vector with components $x_{j}^{k+1} / x_{j}^{k}$ simply by $x^{k+1} / x^{k}$. That these algorithms are indeed reasonable ways to solve the problem (2) will become apparent later in this section. Cross log entropy maximization has been suggested by Herman et al. [26], together with the algorithm

$$
x_{j}^{k+1}=x_{j}^{k}\left\{1+\alpha\left[A^{T} p^{k}\right]_{j=1,2, \ldots, m}-\left[A^{T} q^{k}\right]_{j}\right\}
$$

where $\alpha$ is a relaxation parameter with the same dimension as $x_{j}$. The detailed derivation is given for convergence in [28]. The entropy maximization provides the points that the negative $\log$ energy never increases. The background estimation is done by using the low-pass filter. The high frequency suppressed region provides the background in the images. The orientation filtering is now again applied for the rain removal. This process provides the complete rain removal in the image.

\section{RESULTS AND DISCUSSION}

The proposed method was implemented in MATLAB on a personal computer equipped with Intel Core i5-540M processor and 4-GB memory. The comparative result analysis is provided in table 1 . The baseline 1 and baseline 2 method comparison is performed with the proposed in terms of PSNR and universal image quality index (UIQI). The feature based method gives the result shown in fig 3 and fig 4 that is comparatively complex as compared to decomposition method.

Table 1. Result comparison for the baseline [16], baseline [23], and proposed method

\begin{tabular}{|c|c|c|c|}
\hline Image & Method & PSNR & UIQI \\
\hline \multirow{3}{*}{ Street Image } & Baseline 1 & 31.76 & 0.87 \\
\cline { 2 - 4 } & Baseline 2 & 32.36 & 0.88 \\
\cline { 2 - 4 } & Proposed & 33.34 & 0.93 \\
\hline \multirow{3}{*}{ Tree Image } & Baseline 1 & 31.26 & 0.86 \\
\cline { 2 - 4 } & Baseline 2 & 31.87 & 0.89 \\
\cline { 2 - 4 } & Proposed & 32.89 & 0.91 \\
\hline
\end{tabular}

The decomposition method gives clearer image quality in its characteristics but takes more computational cost in terms of time and input-output transformation. Figure 3 and 4 provides the Original image with rain in (a), the rain removed by baseline 1 [16] method in (b), the rain removed baseline 2 [23] method in (c) and (d) provides the rain removed image by proposed method.

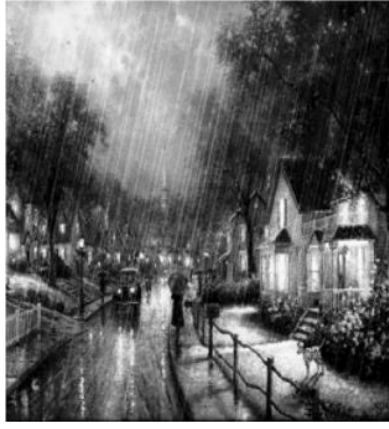

(a)

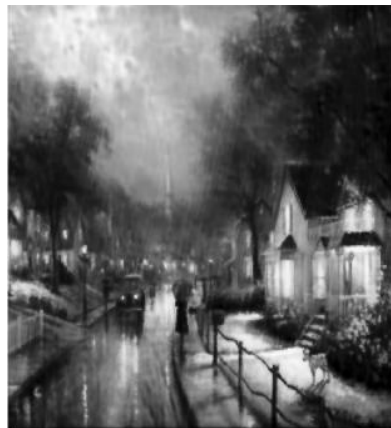

(c)

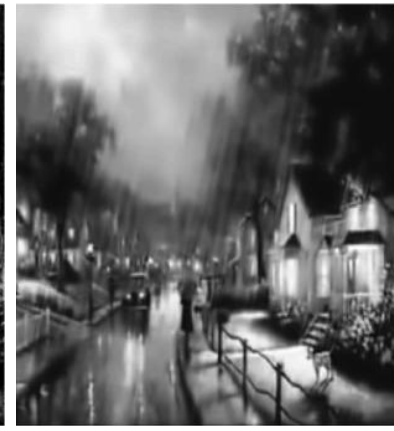

(b)

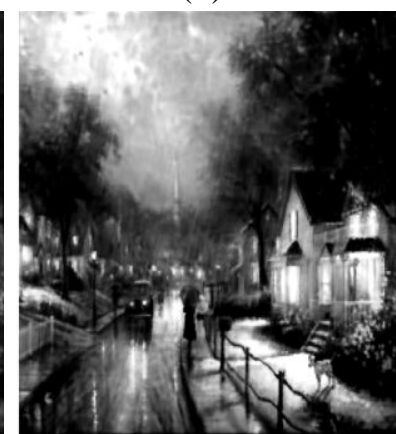

(d)
Fig. 3: (a) Original image with rain (b) rain removed by baseline1 [16] method (c) rain removed baseline 2 [23] method (d) Rain removed image by proposed method.

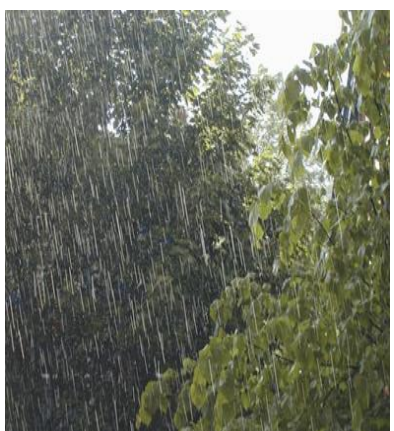

(a)

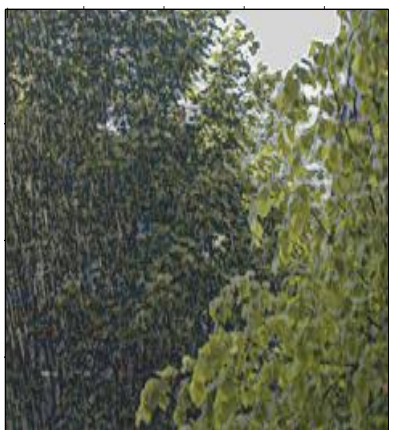

(c)

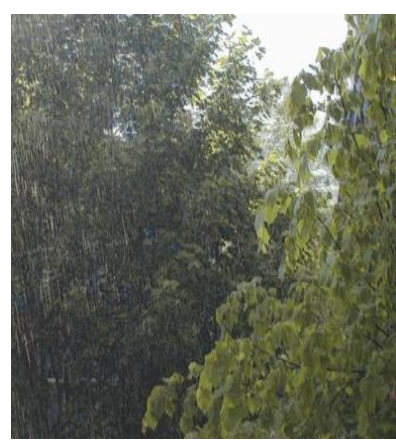

(b)

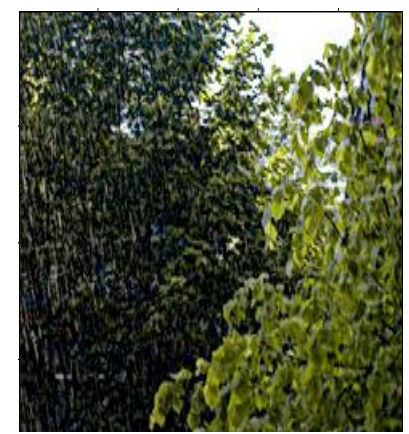

(d)
Fig 4 (a) Original image with rain (b) rain removed by baseline1 [16] method (c) rain removed baseline 2 [23] method (d) Rain removed image by proposed method.

\section{CONCLUSION}

This method is based on single-image rain removal framework. The raindrops are greatly differing from the background, as the intensity of rain drops is higher the background. The entropy maximization is very much suitable 
for the rain removal. Experimental results express the efficacy of the rain removal by proposed algorithm is better than the method based on saturation and visibility features. The table11 provides the quantitative analysis, of result. The removal framework for rain removal is an MCA-based image decomposition problem solved by performing sparse coding and dictionary learning algorithms. This also provided an optional scheme to further enhance the performance of rain removal by introducing an extended dictionary of nonrain atoms learned from nonrain training images.

\section{REFERENCES}

[1] P. C. Barnum, S. Narasimhan, and T. Kanade, "Analysis of rain and snow in frequency space," Int. J. Comput. Vis., vol. 86, no. 2/3, pp. 256-274, Jan. 2010.

[2] K. Garg and S. K. Nayar, "Detection and removal of rain from videos," in Proc. IEEE Conf. Comput. Vis. Pattern Recognit., Jun. 2004, vol. 1, pp. 528-535.

[3] K. Garg and S. K. Nayar, "When does a camera see rain?," in Proc. IEEE Int. Conf. Comput. Vis., Oct. 2005, vol. 2, pp. 1067-1074.

[4] K. Garg and S. K. Nayar, "Vision and rain," Int. J. Comput. Vis., vol. 75, no. 1, pp. 3-27, Oct. 2007.

[5] X. Zhang, H. Li, Y. Qi, W. K. Leow, and T. K. Ng, "Rain removal in video by combining temporal and chromatic properties," in Proc. IEEE Int. Conf. Multimedia Expo., Toronto, ON, Canada, Jul. 2006, pp. 461-464.

[6] N. Brewer and N. Liu, "Using the shape characteristics of rain to identify and remove rain from video," Lecture Notes Comput. Sci., vol. 5342/2008, pp. 451-458, 2008.

[7] J. Bossu, N. Hautière, and J. P. Tarel, "Rain or snow detection in image sequences through use of a histogram of orientation of streaks," Int. J. Comput. Vis., vol. 93, no. 3, pp. 348-367, Jul. 2011.

[8] M. Roser and A. Geiger, "Video-based raindrop detection for improved image registration," in IEEE Int. Conf. Comput. Vis.Workshops, Kyoto, Sep. 2009, pp. 570-577.

[9] J. C. Halimeh and M. Roser, "Raindrop detection on car windshields using geometric-photometric environment construction and intensitybased correlation," in Proc. IEEE Intell. Veh. Symp., Xi'an, China, Jun. 2009, pp. 610-615.

[10] N. Dalal and B. Triggs, "Histograms of oriented gradients for human detection," in Proc. IEEE Conf. Comput. Vis. Pattern Recognit., San Diego, CA, Jun. 2005, vol. 1, pp. 886-893.

[11] J. M. Fadili, J. L. Starck, J. Bobin, and Y. Moudden, "Image decomposition and separation using sparse representations: An overview," Proc.IEEE, vol. 98, no. 6, pp. 983-994, Jun. 2010.

[12] J. L. Starck, M. Elad, and D. L. Donoho, "Image decomposition via the combination of sparse representations and a variational approach," IEEE Trans. Image Process., vol. 14, no. 10, pp. 1570-1582, Oct. 2005.

[13] S. Mallat and Z. Zhang, "Matching pursuits with timefrequency dictionaries," IEEE Trans. Signal Process., vol. 41, no. 12, pp. 3397-3415, Dec. 1993.
[14] D. L. Donoho, "Compressed sensing," IEEE Trans. Inf. Theory, vol. 52, no. 4, pp. 1289-1306, Apr. 2006.

[15] A. M. Bruckstein, D. L. Donoho, and M. Elad, "From sparse solutions of systems of equations to sparse modeling of signals and images," SIAM Rev., vol. 51, no. 1, pp. 34-81, Feb. 2009.

[16] Li-Wei Kang, Chia-Wen Lin and Yu-Hsiang Fu "Automatic single-image-based rain streaks removal via image decomposition IEEE Trans. on Image Process., vol. 21, no. 4, April 2012.

[17] D. Sharma, Y. Kurmi, and V. Chaurasia, "Formation of super- resolution image: a review," Int. Jour. of Emerging Tech. and Adv. Engg., Apr. 2014, vol. 4, no. 4, pp. 218-221.

[18] Y. Kurmi and V. Chaurasia, "An image fusion approach based on adaptive fuzzy logic model with local level processing," Int. Jour. of Comp. Appl., Aug. 2015, vol. 124 , no.1, pp. 39-42.

[19] S. Tiwari, K. Chauhan, and Y. Kurmi "Shadow detection and compensation in aerial images using MATLAB," Int. Jour. of Comp. Appl., June 2015, vol. 119, no.20, pp. 59.

[20] Y. Kurmi and V. Chaurasia, "Performance of haze removal filter for hazy and noisy images," Int. Jour. of Sci. Engg. and Tech., Apr. 2014, vol. 3 no. 4, pp. 437 439.

[21] M. K. Patle, B. Chourasia, and Y. Kurmi, "High dynamic range image analysis through various tone mapping techniques," Int. Jour. of Comp. Appl., vol.153, no. 11, Nov. 2016 pp. 14-17.

[22] A. Kumar, B. Chourasia, and Y. Kurmi, "Image defogging by multiscale depth fusion and hybrid scattering model," International Journal of Computer Applications (0975 - 8887), vol. 155, no 11, Dec. 2016, pp. 34-38.

[23] Soo-Chang Pei, Yu-Tai Tsai, and Chen-Yu Lee "Removing rain and snow in a single image using saturation and visibility features, Graduate Inst. of Comm. Engg., Nat. Taiwan University, Taipei, Taiwan.

[24] Google Goggles [Online]. Available: http://www.google.com/mobile/goggles/

[25] Y. Niu, X. Wu, and G. Shi, "Image enhancement by entropy maximization and quantization resolution upconversion," IEEE Trans. on Image Process., vol. 25, no. 10, Oct. 20164815

[26] G. T. Herman et al., "Maximum a posteriori image reconstruction from projections," in image models (and their speech model cousins), S. Levinson and L. A. Shepp, Eds. New York, NY: Springer-Verlag, 1996.

[27] A. Mishra, B. Chaurasia and Y. Kurmi, "Comparative Analysis of Single Image Shadow Detection and Removal in Aerial Images," International Journal of Advanced Engineering and Management, Vol. 2, No. 4, pp. 86-89, 2017

[28] Y. Cao, P. P. B. Eggermont, and S. Terebey, "Cross burg entropy maximization and its application to ringing suppression in image reconstruction," IEEE Trans. on Image Process., vol. 8, no. 2, Feb. 1999, pp.286-292. 